\title{
ON THE LIMIT CYCLES OF LINEAR DIFFERENTIAL SYSTEMS WITH HOMOGENEOUS NONLINEARITIES
}

\author{
JAUME LLIBRE ${ }^{1}$ AND XIANG ZHANG ${ }^{2}$
}

\begin{abstract}
We consider the class of polynomial differential systems of the form $\dot{x}=\lambda x-y+P_{n}(x, y), \dot{y}=x+\lambda y+Q_{n}(x, y)$, where $P_{n}$ and $Q_{n}$ are homogeneous polynomials of degree $n$. For this class of differential systems we summarize the known results for the existence of limit cycles, and we provide new results for their non-existence and existence.
\end{abstract}

\section{InTRODUCTION AND STATEMENT OF THE MAIN RESULTS}

One of the main problems in the qualitative theory of real planar differential systems is to control the existence, non-existence or uniqueness of limit cycles for a given class of polynomial differential systems.

Limit cycles of planar differential systems were defined by Poincaré [13] and started to be studied intensively at the end of the 1920s by van der Pol [14], Liénard [7] and Andronov [1].

In this work we study the real planar polynomial differential systems of the form

$$
\begin{aligned}
& \dot{x}=\lambda x-y+P_{n}(x, y), \\
& \dot{y}=x+\lambda y++Q_{n}(x, y),
\end{aligned}
$$

where $P_{n}$ and $Q_{n}$ are homogeneous polynomials of degree $n$.

In order to be more precise we need to introduce some notations and basic results. Then, in polar coordinates $(r, \theta)$ defined by $x=$ $r \cos \theta, y=r \sin \theta$, system (1) writes as

$$
\begin{aligned}
& \dot{r}=\lambda r+f(\theta) r^{n}, \\
& \dot{\theta}=1+g(\theta) r^{n-1},
\end{aligned}
$$

where

$$
\begin{aligned}
& f(\theta)=\cos \theta P_{n}(\cos \theta, \sin \theta)+\sin \theta Q_{n}(\cos \theta, \sin \theta), \\
& g(\theta)=\cos \theta Q_{n}(\cos \theta, \sin \theta)-\sin \theta P_{n}(\cos \theta, \sin \theta),
\end{aligned}
$$

2010 Mathematics Subject Classification. 34C35; 34D30.

Key words and phrases. Polynomial differential system, limit cycles, differential equations on the cylinder. 
are homogeneous polynomials of degree $n+1$ in the variables $\cos \theta$ and $\sin \theta$. In the region

$$
C=\left\{(r, \theta): 1+g(\theta) r^{n-1}>0\right\}
$$

the differential system (2) is equivalent to the differential equation

$$
\frac{d r}{d \theta}=\frac{\lambda r+f(\theta) r^{n}}{1+g(\theta) r^{n-1}} .
$$

It is known that the periodic orbits surrounding the origin of system (2) do not intersect the curve $\dot{\theta}=0$ (see the Appendix of [3]). Therefore, these periodic orbits are contained in the region $C$, and consequently also they are periodic orbits of equation (3). Moreover these periodic orbits can be studied doing the change of variables

$$
\rho=\frac{r^{n-1}}{1+g(\theta) r^{n-1}}
$$

due to Cherkas [4], which in fact goes back to Liouville [9]. In the new variable $\rho$ the differential equation (3) writes

(4) $\frac{d \rho}{d \theta}=(n-1) g(\lambda g-f) \rho^{3}+\left((n-1)(f-2 \lambda g)-g^{\prime}\right) \rho^{2}+(n-1) \lambda \rho$.

There are previous results on the existence of limit cycles for the polynomial differential systems (1), using the differential equations (3) or (4), that we summarize in what follows.

(i) If the trigonometric polynomial $f-\lambda g \not \equiv 0$ does not change sign, then system (1) has at most one limit cycle, and when it exists surrounds the origin (see Theorem A of [5]).

(ii) If the trigonometric polynomial $(n-1) g(\lambda g-f) \not \equiv 0$ does not change sign, then equation (1) has at most one limit cycle in the region $r>0$ if $n$ is even, and at most two limit cycles in the region $r>0$ if $n$ is odd, and when they exist surround the origin (see statement (b) of Theorem 1.1 of [2]).

(iii) If the trigonometric polynomial $(n-1)(f-2 \lambda g)-g^{\prime} \not \equiv 0$ does not change sign, then equation (1) has at most two limit cycles, and when they exist surround the origin (see statement (a) of Theorem $\mathrm{C}$ of $[6])$.

(iv) If either the trigonometric polynomial $(n-1) g(\lambda g-f) \equiv 0$ or $(n-1)(f-2 \lambda g)-g^{\prime} \equiv 0$, then equation (1) has at most one limit cycle, and when it exists surrounds the origin (see statement (b) of Theorem C of [6]). 
We remark that all the previous results only provide information on the limit cycles surrounding the origin of the polynomial differential system (1).

We must mention that the first results that finally ended in the result (ii) were given by Pliss [12] and Lins Neto [8].

All results (i)-(iv) are about existence of limit cycles for the polynomial differential systems (1). Now we provide results on the nonexistence of limit cycles for systems (1) and a new result on the existence and uniqueness.

Theorem 1. The polynomial differential system (1) with $n \geq 2$ has no limit cycles surrounding the origin if one of the following condition hold:

(i) if $f=0$;

(ii) if $f-\lambda g=0$;

(iii) if $g=0$;

(iv) if $(2 n-1)^{2} f-\lambda(2 n-3)^{2} g=0$;

(v) if $(f-\lambda g)\left(n^{2} f-\lambda(n-2)^{2} g\right) \leq 0$ for all $\theta$;

(vi) if $(f-\lambda g)\left((2 n-1)^{2} f-\lambda(2 n-3)^{2} g\right) \leq 0$ for all $\theta$.

The polynomial differential system (1) has at most one limit cycle surrounding the origin if the following conditions holds:

(vii) if $(f-\lambda g)\left((2 n-3)^{2} f-\lambda(2 n-1)^{2} g\right) \leq 0$ for all $\theta$.

Theorem 1 is proved in section 3 .

In some sense Theorem 1 is an extension to any positive integer $n$, of a similar result for $n=2$, see Theorem 1 of [10].

\section{Preliminary Results}

In this section we recall some basic results that we shall need for proving our Theorem 1.

The next two results correspond to Theorems 2 and 3 of Lloyd [11].

Lemma 2. We have a differential system in polar coordinates

$$
\begin{aligned}
& \dot{r}=F(r, \theta), \\
& \dot{\theta}=G(r, \theta),
\end{aligned}
$$

defined in a simply connected open set $U$ containing the origin, where $F$ and $G$ are $C^{1} 2 \pi$-periodic functions such that $F(0, \theta)=0$ for all $\theta$, and $G(r, \theta)>0$ in $U$. Then, in $U$ the differential system (5) is equivalent to the differential equation

$$
\frac{d r}{d \theta}=\frac{F(r, \theta)}{G(r, \theta)}=S(r, \theta)
$$


Therefore, if

$$
\frac{\partial S}{\partial r} \not \equiv 0
$$

and

$$
\text { either } \frac{\partial S}{\partial r} \leq 0, \quad \text { or } \frac{\partial S}{\partial r} \geq 0 \quad \text { in } U,
$$

the differential system (5) has no limit cycles in $U$.

Remark 3. We note that in [11] the inequalities (8) appear without the equal, but checking the proof of Theorem 2 of [11] we see that it also works under the conditions (7) and (8).

Lemma 4. Consider the differential system (5) defined in an annular region $\mathcal{A}$ which encircles the origin and where $G(r, \theta)>0$. Then, in $\mathcal{A}$ the differential system (5) is equivalent to the differential equation (6). If (7) and (8) hold in $\mathcal{A}$, then the differential system (5) has at most 1 limit cycle in $\mathcal{A}$.

The remark 3 applies to Lemma 4 but now using the proof of Theorem 3 of [11].

Lemma 5. Under the assumptions of Lemma 4 if $\partial^{3} S / \partial r^{3} \geq 0$ in $\mathcal{A}$, then the differential system (5) has at most 3 limit cycles in $\mathcal{A}$.

Again Remark 3 applies to Lemma 5 but now using the proof of Theorem 8 of [11].

\section{Proof of Theorem 1}

We prove Theorem 1 statement by statement. The proof of the first two statements of Theorem 1 are essentially the same that in the particular case that $n=2$ done in [10], but since are shorter and easier we provide them here for completeness.

Proof of statement $(i)$ of Theorem 1. Since $f=0$ then $d r / d \theta$ does not change sign. If $\lambda \neq 0$ the solutions $r(\theta)$ of (3) increases or decreases, so these solutions cannot be periodic in the region $C$, and consequently the polynomial differential system (1) has no limit cycles surrounding the origin.

If $\lambda=0$ then $d r / d \theta \equiv 0$ and all the solutions in the region $C$ are periodic and circular (except the equilibrium point at the origin), so the system has no isolated periodic orbits surrounding the origin, i.e. no limit cycles surrounding the origin. So statement $(i)$ is proved. 
Proof of statement (ii) of Theorem 1. Since $f-\lambda g=0$ we have that $d r / d \theta=\lambda r$. Now the proof ends following the same arguments than in the proof of statement $(i)$.

Proof of statement (iii) of Theorem 1. If $g=0$ then the differential equation (3) becomes $d r / d \theta=\lambda r+f r^{n}$. Its general solution $r(\theta)$ satisfying that $r(0)=r_{0}>0$ is

$$
r(\theta)=\left(e^{(1-n) \theta \lambda} r_{0}^{-n}\left(r_{0}+(1-n)\left(\int_{0}^{\theta} e^{(n-1) s \lambda} f(s) d s\right) r_{0}^{n}\right)\right)^{\frac{1}{1-n}} .
$$

There is at most a unique value of $r_{0}>0$ such that $r(2 \pi)=r_{0}$ if exists, namely

$$
r_{0}=\left(\frac{e^{2(1-n) \pi \lambda}(n-1) \int_{0}^{2 \pi} e^{(n-1) s \lambda} f(s) d s}{e^{2(1-n) \pi \lambda}-1}\right)^{\frac{1}{1-n}} .
$$

This completes the proof of statement (iii).

Proof of statement (iv) of Theorem 1. From $(2 n-1)^{2} f-\lambda(2 n-3)^{2} g=$ 0 we have $f=\lambda(2 n-3)^{2} g /(2 n-1)^{2}$. Substituting $f$ into the differential equation (3) we get

$$
\frac{d r}{d \theta}=\lambda \frac{r+(2 n-3)^{2} g r^{n} /(2 n-1)^{2}}{1+g r^{n-1}}=S(r, \theta) .
$$

defined in the simply connected region $C$.

We have

$$
\frac{\partial S}{\partial r}=\frac{\lambda\left((2 n-3)^{2} g^{2} r^{2 n-2}+2 g r^{n-1}+(2 n-1)^{2}\right)}{(2 n-1)^{2}\left(1+g r^{n-1}\right)^{2}} .
$$

Then, since $4 g^{2}-4(2 n-1)^{2}(2 n-3)^{2} g^{2}=-g^{2}(n-1)^{2}(1+2 n(n-$ $2)) \leq 0$, we have that $\partial S / \partial R$ satisfies $(7)$ and (8) in the region $S$, and consequently, we can apply Lemma 2 , to the differential equation (9), and the proof of statement $(i v)$ is done.

Proof of statement $(v)$ of Theorem 1. Consider the differential equation (3)

$$
\frac{d r}{d \theta}=\frac{\lambda r+f r^{n}}{1+g r^{n-1}}=S(r, \theta)
$$

defined in the simply connected region $C$.

We have

$$
\frac{\partial S}{\partial r}=\frac{f g r^{2 n-2}+(n f-(n-2) \lambda g) r^{n-1}+\lambda}{\left(1+g r^{n-1}\right)^{2}}
$$


Then, if $(n f-(n-2) \lambda g)^{2}-4 \lambda f g=(f-\lambda g)\left(n^{2} f-\lambda(n-2)^{2} g\right) \leq 0$, we have that $\partial S / \partial R$ satisfies (7) and (8) in the region $S$, and consequently, we can apply Lemma 2 , to the differential equation (10), and the proof of statement $(v)$ is done.

Proof of statement (vi) of Theorem 1. Doing the change of variables $R=\sqrt{r}$ in the region $C$, the differential equation (3) becomes

$$
\frac{d R}{d \theta}=\frac{\lambda R+f R^{2 n-1}}{2\left(1+g R^{2 n-2}\right)}=S(R, \theta) .
$$

Clearly the image of the simply connected region $C$ under the map $r \rightarrow \sqrt{r}=R$ is another simply connected region $S$ containing the origin $R=0$.

We have

$$
\frac{\partial S}{\partial R}=\frac{f g R^{4 n-4}+((2 n-1) f+\lambda(3-2 n) g) R^{2 n-2}+\lambda}{2\left(1+g R^{2 n-2}\right)^{2}} .
$$

Then, if $((2 n-1) f+\lambda(3-2 n) g)^{2}-4 f g \lambda=(f-\lambda g)\left((2 n-1)^{2} f-\lambda(2 n-\right.$ $\left.3)^{2} g\right) \leq 0$, we have that $\partial S / \partial R$ satisfies (7) and (8) in the region $S$, and consequently, we can apply Lemma 2 , to the differential equation (11), and the proof of statement (vi) follows.

Proof of statement (vii) of Theorem 1. Doing the change of variables $R=1 / \sqrt{r}$ in the region $C$, the differential equation (3) becomes

$$
\frac{d R}{d \theta}=\frac{-f R-\lambda R^{2 n-1}}{2\left(g+R^{2 n-2}\right)}=S(R, \theta) .
$$

Now the image of the region $C$ by the map $r \rightarrow 1 / \sqrt{r}=R$ is an annular region $\mathcal{A}$, and one of the boundaries of this annulus is the infinity.

We get

$$
\frac{\partial S}{\partial R}=-\frac{\lambda R^{4 n-4}+((3-2 n) f+\lambda(2 n-1) g) R^{2 n-2}+f g}{2\left(g+R^{2 n-2}\right)^{2}} .
$$

Then, clearly if $((3-2 n) f+\lambda(2 n-1) g)^{2}-4 \lambda f g=(f-\lambda g)\left((2 n-3)^{2} f-\right.$ $\left.\lambda(2 n-1)^{2} g\right) \leq 0$, we have that $\partial S / \partial R$ satisfies conditions $(7)$ and (8) in the annular region $\mathcal{A}$, and consequently we can apply Lemma 4 , to the differential equation (12), and this completes the proof of statement (vii).

In $[10]$ it is proved that the system

$$
\begin{aligned}
& \dot{x}=\lambda x-y+\frac{1}{5 \lambda}(5 \lambda x-y)^{2}, \\
& \dot{y}=x+\lambda y+\frac{1}{5 \lambda}(5 \lambda x-y)(x+5 \lambda y),
\end{aligned}
$$


has a unique limit cycle surrounding the origin. We note that this system satisfies the assumption (vii) of Theorem 1 for $n=2$.

\section{ACKNOWLEDGMENTS}

The first author is partially supported by a MINECO/FEDER grant MTM2008-03437, a CIRIT grant number 2009SGR-410, an ICREA Academia, two grants FP7-PEOPLE-2012-IRSES 316338 and 318999, the grant UNAB13-4E-1604, and from the recruitment program of high-end foreign experts of China.

The second author is partially supported by NNSF of China grant number 11271252, by RFDP of Higher Education of China grant number 20110073110054, and by FP7-PEOPLE-2012-IRSES-316338 of Europe, and by innovation program of Shanghai municipal education commission grant 15ZZ012.

This work was done during the visit of the first author to Shanghai Jiao Tong University, who appreciate its support and hospitality.

\section{REFERENCES}

[1] A.A. Andronov, Les cycles limites de Poincaré et la théorie des oscillations auto-entretenues, C.R. Acad. Sci. Paris 89 (1929), 559-561.

[2] M. Carbonell AND J. Llibre, Limit cycles of a class of polynomial systems, Proc. Royal Soc. Edinburgh 109A (1988), 187-199.

[3] M. Carbonell And J. Llibre, in Hopf bifurcation, averaging methods and Liapunov quantities for polynomial systems with homogeneous nonlinearities, Proc. European Conference on Iteration Theory, ECIT87, World Scientific, Singapore, 1989, pp. 145-160.

[4] L.A. Cherkas, Number of limit cycles of an autonomous second-order system, Differential Equations 5 (1976), 666-668.

[5] T. Coll, A. Gasull and R. Prohens, Differential equations defined by the sum of two quasi-homogeneous vector fields, Can. J. Math. 49 (1997), 212-231.

[6] A. Gasull And J. LliBre, Limit cycles for a class of Abel equations, SIAM J. Math. Anal. 21 (1990), 1235-1244.

[7] A. LiÉnARD, Etude des oscillations entretenues, Rev. Générale de l'Electricité 23 (1928), 901-912.

[8] A. Lins Neto, On the number of solutions of the equation $d x / d t=$ $\sum_{j=0}^{n} a_{j}(t) x^{j}, 0 \leq t \leq 1$, for which $x(0)=x(1)$, Invent. Math. 59 (1980), $67-76$.

[9] R. Liouville, Sur une équation différentielle du premier ordre, (French) Acta Math. 27 (1903), 55-78.

[10] J. Llibre AND X. ZHANG, Non-existence, existence and uniqueness of limit cycles for quadratic polynomial differential systems, preprint, 2014.

[11] N.G. LlOYD, A note on the number of limit cycles in certain two-dimensional systems, J. London Math. Soc. 20 (1979), 277-286.

[12] V.A. Pliss, Non-local problems of the Theory of Oscillations, Academic Press, New York, 1966 
[13] H. Poincaré, Mémoire sur les courbes définies par une équation differentielle I, II, J. Math. Pures Appl. 7 (1881), 375-422; 8 (1882), 251-296; Sur les courbes définies pas les équations differentielles III, IV, 1 (1885), 167-244; 2 (1886), 155-217.

[14] VAN DER POL, On relaxation-oscillations, Phil. Mag. 2 (1926), 978-992.

${ }^{1}$ Departament de Matemàtiques, Universitat Autònoma de Barcelona, 08193 Bellaterra, Barcelona, Catalonia, Spain

E-mail address: jllibre@mat.uab.cat

3 Department of Mathematics, MOE-LSC, Shanghai Jiao tong University, Shanghai, 200240, P. R. China

E-mail address: xzhang@sjtu.edu.cn 